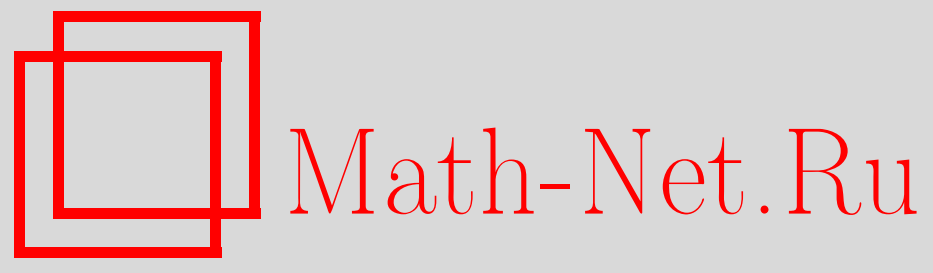

Ф. Г. Хуштова, Фундаментальное решение модельного уравнения аномальной диффузии дробного порядка, Вестн. Сам. гос. техн. ун-та. Сер. Физ.-мат. науки, 2015, номер 4, 722-735

DOI: https://doi.org/10.14498/vsgtu1445

Использование Общероссийского математического портала MathNet.Ru подразумевает, что вы прочитали и согласны с пользовательским соглашением

http://www. mathnet.ru/rus/agreement

Параметры загрузки:

IP: 3.80 .253 .173

26 апреля 2023 г., 15:55:23

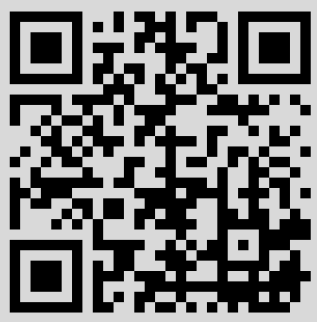


УДК 517.968.7

\title{
ФУНДАМЕНТАЛЬНОЕ РЕШЕНИЕ МОДЕЛЬНОГО УРАВНЕНИЯ АНОМАЛЬНОЙ ДИФФУЗИИ ДРОБНОГО ПОРЯДКА
}

\section{Ф. Г. Хуитова}

Институт прикладной математики и автоматизации, Россия, 360000, Нальчик, ул. Шортанова, 89 а.

\begin{abstract}
Аннотация
Построено фундаментальное решение модельного уравнения аномальной диффузии с оператором дробного дифференцирования РиманаЛиувилля. С использованием свойств интегрального преобразования с функцией Райта в ядре, приведены оценки для фундаментального решения. Показано, что когда исследуемое уравнение переходит в уравнение диффузии дробного порядка, построенное фундаментальное решение переходит в соответствующее фундаментальное решение для уравнения диффузии дробного порядка. Построено общее представление решения рассматриваемого уравнения в прямоугольной области.
\end{abstract}

Ключевые слова: аномальная диффузия, диффузия дробного порядка, оператор Римана-Лиувилля, фундаментальное решение, общее представление решения, модифицированная функция Бесселя, функция Райта, интегральное преобразование с функцией Райта в ядре.

doi: http://dx.doi.org/10.14498/vsgtu1445

Введение. Пусть $D_{a y}^{\gamma}$ - оператор интегро-дифференцирования в смысле Римана-Лиувилля дробного порядка $\gamma$ с началом в точке $a$ и с концом в точке y, который определяется следующим образом [1, с. 28] [2, с. 14]:

$$
\begin{gathered}
D_{a y}^{\gamma} g(y)=\frac{\operatorname{sign}(y-a)}{\Gamma(-\gamma)} \int_{a}^{y} \frac{g(t)}{|y-t|^{\gamma+1}} d t, \quad \gamma<0 ; \\
D_{a y}^{\gamma} g(y)=g(y), \quad \gamma=0 ; \\
D_{a y}^{\gamma} g(y)=\operatorname{sign}^{n}(y-a) \frac{d^{n}}{d y^{n}} D_{a y}^{\gamma-n} g(y), \quad n-1<\gamma \leqslant n, n \in \mathbb{N} .
\end{gathered}
$$

Здесь $\Gamma(s)$ - гамма-функция Эйлера.

В области $\Omega=\left\{(x, y): r_{1}<x<r_{2}, 0<y<T\right\}$ рассмотрим уравнение

$$
\mathbf{L} u(x, y) \equiv|x|^{-b} \frac{\partial}{\partial x}\left(|x|^{b} \frac{\partial}{\partial x} u(x, y)\right)-D_{0 y}^{\alpha} u(x, y)=f(x, y)
$$

(C) 2015 Самарский государственный технический университет.

\section{Образец для цитирования}

Хуш т в в $Ф . Г$. Фундаментальное решение модельного уравнения аномальной диффузии дробного порядка // Вестн. Сам. гос. техн. ун-та. Сер. Физ.-мат. науки, 2015. Т. 19, № 4. C. $722-735$. doi: $10.14498 /$ vsgtu1445.

\section{Сведения об авторе}

Фатима Гидовна Хуштова (khushtova@yandex.ru), научный сотрудник, отдел САПР смешанных систем и управления. 
где $|b|<1,0<\alpha \leqslant 1$.

Пусть $\Omega^{+}=\Omega \cap\{x>0\}, \Omega^{-}=\Omega \cap\{x<0\}, \bar{\Omega}$ - замыкание области $\Omega$.

ОПРедЕлениЕ. Регулярным решением уравнения (1) в области $\Omega$ назовем функцию $u=u(x, y)$, удовлетворяющую уравнению $(1)$ в области $\Omega^{+} \cup \Omega^{-}$, и такую, что $y^{1-\alpha} u \in C(\bar{\Omega}),|x|^{b} u_{x} \in C(\Omega), u_{x x}, D_{0 y}^{\alpha} u \in C\left(\Omega^{+} \cup \Omega^{-}\right)$.

Уравнение $(1)$ при $\alpha=1, f(x, y) \equiv 0$ обращается в уравнение

$$
u_{x x}(x, y)+\frac{b}{x} u_{x}(x, y)-u_{y}(x, y)=0,
$$

которое при $x>0$ было объектом исследования работы [3]. Уравнение (2) с помощью замены $|\xi|=[|x| /(1-b)]^{1-b}$ сводится к уравнению

$$
|\xi|^{q} u_{\xi \xi}(\xi, y)-u_{y}(\xi, y)=0, \quad q=2 b /(b-1)
$$

и оно при $q<1$ было исследовано в работе [4], а при $q=1$ - в работах [5,6].

В случае, когда $b=0,0<\alpha<2$, уравнение (1) совпадает с диффузионноволновым уравнением, исследованным в работах многих авторов. Например, в работах $[7,8]$ в терминах функции Райта построено фундаментальное решение диффузионно-волнового уравнения с оператором Римана-Лиувилля, в работе [9] построено фундаментальное решение многомерного диффузионноволнового уравнения с оператором Джрбашяна-Нерсесяна. В работах $[10,11]$ методом интегральных преобразований исследована задача Коши для более общих диффузионных и волновых уравнений дробного порядка с производной Римана-Лиувилля. Решения исследуемых задач выписаны в терминах $H$-функции Фокса и функции типа Райта. В работах $[12,13]$ исследовалась задача Коши для уравнения диффузии дробного порядка с регуляризованной дробной производной.

В работе А. В. Псху [2] методом функции Грина построены решения первой, второй и смешанной краевых задач в прямоугольной области и решена задача Коши для диффузионно-волнового уравнения. Более полную библиографию работ, посвященных таким уравнениям, можно найти также в [2].

Уравнение вида (1), а именно уравнение

$$
D_{0 t}^{2 / d_{w}} P(r, t)=\frac{1}{r^{d_{s}-1}} \frac{\partial}{\partial r}\left(r^{d_{s}-1} \frac{\partial P(r, t)}{\partial r}\right),
$$

где $d_{w}$ и $d_{s}$ характеризуют фрактальную размерность среды, $P(r, t)$ - плотность пространственного распределения частиц в момент времени $t$, было предложено в работе [14] для описания процессов переноса в средах, имеющих фрактальную размерность.

Интерес к изучению уравнения (1) вызван также его приложениями при решении задач физики, астрономии и других прикладных наук [15-19].

1. Некоторые специальные функции. Приведем некоторые свойства двух специальных функций, которые понадобятся для дальнейшего изложения.

ОПРЕДЕЛЕНиЕ. Функция $I_{\nu}(z)$, определяемая рядом

$$
I_{\nu}(z)=\sum_{n=0}^{\infty} \frac{1}{n ! \Gamma(\nu+n+1)}\left(\frac{z}{2}\right)^{\nu+2 n},
$$


называется модифищированной иилиндрической или бесселевой функицей первого рода порядка $\nu[20,21]$.

При $|\arg z| \leqslant \pi / 2-\varepsilon$, где $\varepsilon-$ произвольное малое положительное число, и $|z| \rightarrow \infty$ справедлива асимптотическая формула

$$
I_{\nu}(z)=\frac{e^{z}}{\sqrt{2 \pi z}}\left[1+O\left(z^{-1}\right)\right]
$$

Имеют место следующие формулы дифференцирования:

$$
\begin{gathered}
\frac{d}{d z}\left[z^{\nu} I_{\nu}(z)\right]=z^{\nu} I_{\nu-1}(z), \\
\frac{d}{d z}\left[z^{-\nu} I_{\nu}(z)\right]=z^{-\nu} I_{\nu+1}(z),
\end{gathered}
$$

откуда вытекают рекуррентные формулы

$$
\begin{aligned}
& z I_{\nu}^{\prime}(z)+\nu I_{\nu}(z)=z I_{\nu-1}(z), \\
& z I_{\nu}^{\prime}(z)-\nu I_{\nu}(z)=z I_{\nu+1}(z) .
\end{aligned}
$$

Модифицированная цилиндрическая функция $I_{\nu}(z)$ порядков $\nu=1 / 2$ и $\nu=-1 / 2$ выражается через элементарные функции

$$
I_{1 / 2}(z)=\frac{e^{z}-e^{-z}}{\sqrt{2 \pi z}}, \quad I_{-1 / 2}(z)=\frac{e^{z}+e^{-z}}{\sqrt{2 \pi z}} .
$$

ОПРедЕлЕНиЕ. Функция $\phi(\rho, \delta ; z)$, определяемая рядом

$$
\phi(\rho, \delta ; z)=\sum_{n=0}^{\infty} \frac{z^{n}}{n ! \Gamma(\rho n+\delta)}, \quad \rho>-1,
$$

называется функиией Райта [22, 23].

При $\rho=-1 / 2$ и $\delta=1 / 2$ функция $\phi(\rho, \delta ; z)$ выражается через экспоненциальную функцию по формуле

$$
\sqrt{\pi} \phi\left(-\frac{1}{2}, \frac{1}{2} ;-z\right)=e^{-z^{2} / 4}
$$

2. Интегральное преобразование с функцией Райта в ядре. В работе $[2$, c. 72] определено интегральное преобразование для функции $v(y)$, заданной на положительной полуоси

$$
A^{\alpha, \mu} v(y) \equiv\left(A^{\alpha, \mu} v\right)(y)=\int_{0}^{\infty} v(t) y^{\mu-1} \phi\left(-\alpha, \mu ;-t y^{-\alpha}\right) d t, \quad 0<\alpha<1 .
$$

В случае, когда $\mu=0$, введено обозначение $A^{\alpha, 0} v(y)=A^{\alpha} v(y)$. Если преобразование $A^{\alpha, \mu}$ применяется к функции, зависящей от нескольких переменных, то в случае необходимости с помощью нижнего индекса обозначается переменная, по которой проводится преобразование. Например, $A_{y}^{\alpha, \mu} v(x, y)$. 
Интеграл (11) будет сходиться, если функция $v(y)$ интегрируема на любом конечном отрезке положительной полуоси и выполняются асимптотические неравенства

$$
\begin{array}{ll}
|v(y)|<c y^{\lambda}, & \lambda>-1, \mu \neq 0 ; \lambda>-2, \mu=0, \quad y \rightarrow 0 \\
|v(y)|<c \exp \left(k y^{\lambda}\right), & \lambda<1 /(1-\alpha), \quad y \rightarrow \infty
\end{array}
$$

где $c$ и $k$-положительные постоянные.

Приведем некоторые свойства преобразования $A^{\alpha, \mu}[2$, с. $78-83]$.

$1^{\circ}$. Пусть $v(y)$ непреръвна в точке $y=0$ и дифберениируема при у > 0 . Тогда

$$
D_{0 y}^{\alpha} A^{\alpha, \mu} v(y)=A^{\alpha, \mu} v^{\prime}(y)+\frac{y^{\mu-1}}{\Gamma(\mu)} v(0) .
$$

В частности,

$$
D_{0 y}^{\alpha} A^{\alpha} v(y)=A^{\alpha} v^{\prime}(y)
$$

$2^{\circ}$. Пусть $0 \leqslant \mu \leqslant \alpha u \lim _{y \rightarrow 0} D_{0 y}^{-\mu / \alpha} v(y)=v_{0}<\infty$. Тогда

$$
\lim _{y \rightarrow 0} D_{0 y}^{\alpha-1} A^{\alpha, \mu} v(y)=v_{0}
$$

$3^{\circ}$. Eсли $u(y) \leqslant v(y) u \mu \geqslant 0$, mo

$$
A^{\alpha, \mu} u(y) \leqslant A^{\alpha, \mu} v(y)
$$

Для степенной функции и функции Райта справедливы следующие формулы [2, с. 74, 84]:

$$
\begin{gathered}
A^{\alpha, \mu} y^{\delta-1}=y^{\alpha \delta+\mu-1} \frac{\Gamma(\delta)}{\Gamma(\alpha \delta+\mu)}, \quad \delta>0, \mu \neq 0 ; \quad \delta \neq 0, \mu=0, \\
A^{\alpha, \mu} y^{\delta-1} \phi\left(\rho, \delta ;-c y^{\rho}\right)=y^{\alpha \delta+\mu-1} \phi\left(\alpha \rho, \alpha \delta+\mu ;-c y^{\alpha \rho}\right), \quad \delta>\rho .
\end{gathered}
$$

Здесь $c-$ положительная постоянная.

\section{3. Фундаментальное решение.}

ОПРЕДЕЛЕНиЕ. Функцию $w=w(x, y ; \xi, \eta)$ назовем фундаментальным решением уравнения (1), если

1) для любых фиксированных $\xi$ и $\eta$ функция $w(x, y ; \xi, \eta)$ как функция переменных $x$ и $y, x \neq 0, y>\eta$, удовлетворяет уравнению

$$
|x|^{-b} \frac{\partial}{\partial x}\left(|x|^{b} \frac{\partial}{\partial x} w(x, y ; \xi, \eta)\right)-D_{\eta y}^{\alpha} w(x, y ; \xi, \eta)=0
$$

2) для любой функции $h(x) \in C\left[x_{1} ; x_{2}\right]$ выполняется соотношение

$$
\lim _{\eta \rightarrow y} \int_{x_{1}}^{x_{2}}|\xi|^{b} h(\xi) D_{y \eta}^{\alpha-1} w(x, y ; \xi, \eta) d \xi=h(x), \quad x_{1}<x<x_{2}
$$


Обозначим через

$$
\Gamma(x, \xi, y)=A_{y}^{\alpha} g(x, \xi, y)
$$

где

$$
g(x, \xi, y)=\frac{|x|^{\beta}|\xi|^{\beta}}{4 y} \exp \left(-\frac{x^{2}+\xi^{2}}{4 y}\right)\left[I_{\beta}\left(\frac{|x \xi|}{2 y}\right)+I_{-\beta}\left(\frac{|x \xi|}{2 y}\right)\right], \quad \beta=\frac{1-b}{2} .
$$

Справедливы следующие свойства функции $\Gamma(x, \xi, y)$.

Свойство $1^{\circ}$. Для функиии $\Gamma(x, \xi, y)$ при $|x \xi| \leqslant 2 y$ имеют место следующие ощенки:

$$
\begin{gathered}
|\Gamma(x, \xi, y)| \leqslant \text { const } \cdot y^{\alpha \beta-1}, \\
\left|\frac{\partial^{n+1}}{\partial x^{n+1}} \Gamma(x, \xi, y)\right| \leqslant \text { const } \cdot|x|^{2 \beta-n-1}|\xi|^{2 \beta} y^{-\alpha \beta-1}, \quad \beta \neq 1 / 2, \\
\left|\frac{\partial^{2 n}}{\partial x^{2 n}} \Gamma(x, \xi, y)\right| \leqslant \text { const } \cdot y^{-\alpha(2 n+1) / 2+\alpha-1}, \quad \beta=1 / 2, \\
\left|\frac{\partial^{2 n+1}}{\partial x^{2 n+1}} \Gamma(x, \xi, y)\right| \leqslant \text { const } \cdot|\xi| y^{-\alpha(2 n+1) / 2-1}, \quad \beta=1 / 2, \\
\left|D_{0 y}^{\alpha} \Gamma(x, \xi, y)\right| \leqslant \text { const } \cdot y^{\alpha \beta-\alpha-1},
\end{gathered}
$$

а при $|x \xi|>2 y-$ оценки

$$
\begin{gathered}
\left|\frac{\partial^{n}}{\partial x^{n}} \Gamma(x, \xi, y)\right| \leqslant \text { const } \cdot|x|^{\beta+(2 n-1) / 2}|\xi|^{\beta-1 / 2} A_{y}^{\alpha} y^{-(2 n-1) / 2-1} \exp \left(-\frac{(x-\xi)^{2}}{4 y}\right), \\
\left|D_{0 y}^{\alpha} \Gamma(x, \xi, y)\right| \leqslant \text { const } \cdot|x|^{\beta+3 / 2}|\xi|^{\beta-1 / 2} A_{y}^{\alpha} y^{-5 / 2} \exp \left(-\frac{(x-\xi)^{2}}{4 y}\right),
\end{gathered}
$$

əде $n=0,1,2, \ldots$

Доказательств о. Оценки при $|x \xi| \leqslant 2 y$ следуют из представлений (3), (17), (18), формул дифференцирования (5), (6) для функции $I_{\nu}(z)$, неравенства (14), формулы (15) и $0<e^{-z}<1$ при $z>0$. Для вывода оценок при $|x \xi|>2 y$ вместо (3) нужно использовать асимптотическую формулу (4).

Свойство $2^{\circ}$. Функиия $\Gamma(x, \xi, y)$ при $x \neq 0, y>0$ и фиксированном $\xi$ является решением уравнения

$$
\mathbf{L} \Gamma(x, \xi, y)=0
$$

Доказ ательств в. Действительно, обозначим

$$
\begin{aligned}
& g_{1}(x, \xi, y)=\frac{x^{\beta} \xi^{\beta}}{4 y} \exp \left(-\frac{x^{2}+\xi^{2}}{4 y}\right) I_{\beta}\left(\frac{x \xi}{2 y}\right) \\
& g_{2}(x, \xi, y)=\frac{x^{\beta} \xi^{\beta}}{4 y} \exp \left(-\frac{x^{2}+\xi^{2}}{4 y}\right) I_{-\beta}\left(\frac{x \xi}{2 y}\right) .
\end{aligned}
$$


Считая для упрощения доказательства, что $x>0, \xi>0$, продифференцируем равенство (17) по $x$, используя формулы (5) и (6) при $\nu=\beta$ и $\nu=-\beta$ соответственно. В результате получим

$$
\frac{\partial}{\partial x} \Gamma(x, \xi, y)=A_{y}^{\alpha} \frac{\partial}{\partial x} g(x, \xi, y)
$$

где

$$
\frac{\partial}{\partial x} g(x, \xi, y)=\frac{\partial}{\partial x}\left(g_{1}(x, \xi, y)+g_{2}(x, \xi, y)\right)
$$

$$
\begin{gathered}
\frac{\partial}{\partial x} g_{1}(x, \xi, y)=\left\{\frac{x^{\beta} \xi^{\beta+1}}{2(2 y)^{2}} I_{\beta-1}\left(\frac{x \xi}{2 y}\right)-\frac{x^{\beta+1} \xi^{\beta}}{2(2 y)^{2}} I_{\beta}\left(\frac{x \xi}{2 y}\right)\right\} \exp \left(-\frac{x^{2}+\xi^{2}}{4 y}\right) \\
\frac{\partial}{\partial x} g_{2}(x, \xi, y)=\left\{\frac{x^{\beta} \xi^{\beta+1}}{2(2 y)^{2}} I_{1-\beta}\left(\frac{x \xi}{2 y}\right)-\frac{x^{\beta+1} \xi^{\beta}}{2(2 y)^{2}} I_{-\beta}\left(\frac{x \xi}{2 y}\right)\right\} \exp \left(-\frac{x^{2}+\xi^{2}}{4 y}\right) .
\end{gathered}
$$

Умножим (20) на $x^{1-2 \beta}$ и продифференцируем полученное равенство по $x$, используя формулу (6) при $\nu=\beta-1$. Воспользуемся затем формулой (7) при $\nu=\beta$ и умножим полученное равенство на $x^{2 \beta-1}$. Аналогично поступим и с равенством (21). Умножим его на $x^{1-2 \beta}$ и продифференцируем полученное равенство по $x$, используя формулу (5) при $\nu=1-\beta$. Воспользуемся затем формулой (8) при $\nu=-\beta$ и умножим полученное равенство на $x^{2 \beta-1}$. В итоге, учитывая обозначение $b=1-2 \beta$, получим

$$
x^{-b} \frac{\partial}{\partial x}\left(x^{b} \frac{\partial}{\partial x} \Gamma(x, \xi, y)\right)=A_{y}^{\alpha} x^{-b} \frac{\partial}{\partial x}\left(x^{b} \frac{\partial}{\partial x} g(x, \xi, y)\right),
$$

где

$$
\begin{aligned}
& x^{-b} \frac{\partial}{\partial x}\left(x^{b} \frac{\partial}{\partial x} g(x, \xi, y)\right)=x^{-b} \frac{\partial}{\partial x}\left(x^{b} \frac{\partial}{\partial x}\left(g_{1}(x, \xi, y)+g_{2}(x, \xi, y)\right)\right), \\
& x^{-b} \frac{\partial}{\partial x}\left(x^{b} \frac{\partial}{\partial x} g_{1}(x, \xi, y)\right)=\left\{\frac{x^{\beta+2} \xi^{\beta}}{2(2 y)^{3}} I_{\beta}\left(\frac{x \xi}{2 y}\right)+\frac{x^{\beta} \xi^{\beta+2}}{2(2 y)^{3}} I_{\beta}\left(\frac{x \xi}{2 y}\right)-\right. \\
& \left.-\frac{x^{\beta} \xi^{\beta}}{(2 y)^{2}} I_{\beta}\left(\frac{x \xi}{2 y}\right)-\frac{x^{\beta+1} \xi^{\beta+1}}{(2 y)^{3}} I_{\beta}^{\prime}\left(\frac{x \xi}{2 y}\right)\right\} \exp \left(-\frac{x^{2}+\xi^{2}}{4 y}\right), \\
& x^{-b} \frac{\partial}{\partial x}\left(x^{b} \frac{\partial}{\partial x} g_{2}(x, \xi, y)\right)=\left\{\frac{x^{\beta+2} \xi^{\beta}}{2(2 y)^{3}} I_{-\beta}\left(\frac{x \xi}{2 y}\right)+\frac{x^{\beta} \xi^{\beta+2}}{2(2 y)^{3}} I_{-\beta}\left(\frac{x \xi}{2 y}\right)-\right. \\
& \left.-\frac{x^{\beta} \xi^{\beta}}{(2 y)^{2}} I_{-\beta}\left(\frac{x \xi}{2 y}\right)-\frac{x^{\beta+1} \xi^{\beta+1}}{(2 y)^{3}} I_{-\beta}^{\prime}\left(\frac{x \xi}{2 y}\right)\right\} \exp \left(-\frac{x^{2}+\xi^{2}}{4 y}\right) \text {. }
\end{aligned}
$$

Далее из формулы (12) следует

$$
D_{0 y}^{\alpha} \Gamma(x, \xi, y)=A_{y}^{\alpha} \frac{\partial}{\partial y} g(x, \xi, y),
$$


где

$$
\frac{\partial}{\partial y} g(x, \xi, y)=\frac{\partial}{\partial y}\left(g_{1}(x, \xi, y)+g_{2}(x, \xi, y)\right)
$$

$$
\begin{aligned}
& \frac{\partial}{\partial y} g_{1}(x, \xi, y)=\left\{\frac{x^{\beta+2} \xi^{\beta}}{2(2 y)^{3}} I_{\beta}\left(\frac{x \xi}{2 y}\right)+\frac{x^{\beta} \xi^{\beta+2}}{2(2 y)^{3}} I_{\beta}\left(\frac{x \xi}{2 y}\right)-\right. \\
&\left.\quad-\frac{x^{\beta} \xi^{\beta}}{(2 y)^{2}} I_{\beta}\left(\frac{x \xi}{2 y}\right)-\frac{x^{\beta+1} \xi^{\beta+1}}{(2 y)^{3}} I_{\beta}^{\prime}\left(\frac{x \xi}{2 y}\right)\right\} \exp \left(-\frac{x^{2}+\xi^{2}}{4 y}\right), \\
& \frac{\partial}{\partial y} g_{2}(x, \xi, y)=\left\{\frac{x^{\beta+2} \xi^{\beta}}{2(2 y)^{3}} I_{-\beta}\left(\frac{x \xi}{2 y}\right)+\frac{x^{\beta} \xi^{\beta+2}}{2(2 y)^{3}} I_{-\beta}\left(\frac{x \xi}{2 y}\right)-\right. \\
&\left.-\frac{x^{\beta} \xi^{\beta}}{(2 y)^{2}} I_{-\beta}\left(\frac{x \xi}{2 y}\right)-\frac{x^{\beta+1} \xi^{\beta+1}}{(2 y)^{3}} I_{-\beta}^{\prime}\left(\frac{x \xi}{2 y}\right)\right\} \exp \left(-\frac{x^{2}+\xi^{2}}{4 y}\right) .
\end{aligned}
$$

Подставляя (22) и (23) в уравнение (19), видим, что оно обращается в тождество.

Обозначим через $\Omega_{y}=\left\{(\xi, \eta): r_{1}<\xi<r_{2}, 0<\eta<y\right\}$.

Свойство $3^{\circ}$. В области $\Omega_{y}$ функиия

$$
\Gamma(x, \xi, y-\eta)=\left.A_{t}^{\alpha} g(x, \xi, t)\right|_{t=y-\eta}
$$

при биксированных х и у как функиия переменных $\xi u \eta, \xi \neq 0,0<\eta<y$, является решением сопряженного уравнения

$$
\mathbf{L}^{*} \Gamma(x, \xi, y-\eta) \equiv|\xi|^{-b} \frac{\partial}{\partial \xi}\left(|\xi|^{b} \frac{\partial}{\partial \xi} \Gamma(x, \xi, y-\eta)\right)-D_{y \eta}^{\alpha} \Gamma(x, \xi, y-\eta)=0 .
$$

Свойство $3^{\circ}$ является следствием свойства $2^{\circ}$.

Свойство $4^{\circ}$. Для любой функиии $h(x) \in C\left[x_{1} ; x_{2}\right]$ выполняется соотношение

$$
\lim _{\eta \rightarrow y} \int_{x_{1}}^{x_{2}}|\xi|^{b} h(\xi) D_{y \eta}^{\alpha-1} \Gamma(x, \xi, y-\eta) d \xi=h(x), \quad x_{1}<x<x_{2}
$$

Доказа тель ст в о. Обозначив через $t=y-\eta$, перепишем левую часть равенства (24) в виде

$$
\lim _{\eta \rightarrow y} \int_{x_{1}}^{x_{2}}|\xi|^{b} h(\xi) D_{y \eta}^{\alpha-1} \Gamma(x, \xi, y-\eta) d \xi=\lim _{t \rightarrow 0} \int_{x_{1}}^{x_{2}}|\xi|^{b} h(\xi) D_{0 t}^{\alpha-1} \Gamma(x, \xi, t) d \xi
$$

Тогда в силу формулы (13) можно записать

$$
\lim _{t \rightarrow 0} \int_{x_{1}}^{x_{2}}|\xi|^{b} h(\xi) D_{0 t}^{\alpha-1} \Gamma(x, \xi, t) d \xi=
$$




$$
\begin{aligned}
=\lim _{t \rightarrow 0}\left[\int_{x_{1}}^{x_{2}}|\xi|^{b} g(x, \xi, t)[h(\xi)-h(x)] d \xi+h(x)\right. & \left.\int_{x_{1}}^{x_{2}}|\xi|^{b} g(x, \xi, t) d \xi\right]= \\
& =\lim _{t \rightarrow 0}\left[J_{1}(x, t)+J_{2}(x, t)\right] .
\end{aligned}
$$

Разбивая промежуток интегрирования на части, представим $J_{1}(x, t)$ в виде суммы трех слагаемых:

$$
\begin{aligned}
& J_{1}(x, t)=\int_{x_{1}}^{x-\varepsilon}|\xi|^{b} g(x, \xi, t)[h(\xi)-h(x)] d \xi+ \\
& \quad+\int_{x-\varepsilon}^{x+\varepsilon}|\xi|^{b} g(x, \xi, t)[h(\xi)-h(x)] d \xi+\int_{x+\varepsilon}^{x_{2}}|\xi|^{b} g(x, \xi, t)[h(\xi)-h(x)] d \xi,
\end{aligned}
$$

где $\varepsilon-$ произвольное малое положительное число.

Из (3), (4) и (18) следуют оценки

$$
\begin{gathered}
|g(x, \xi, t)| \leqslant \text { const } \cdot t^{\beta-1}, \quad|x \xi| \leqslant 2 t \\
|g(x, \xi, t)| \leqslant \text { const } \cdot x^{\beta-1 / 2} \xi^{\beta-1 / 2} t^{-1 / 2} \exp \left(-\frac{(x-\xi)^{2}}{4 t}\right), \quad|x \xi|>2 t .
\end{gathered}
$$

В силу оценки (27) первое и третье слагаемые в (25) стремятся к нулю при $t \rightarrow 0$.

Обозначим через $\omega(\varepsilon)=\sup |h(x)-h(\xi)|, \xi \in[x-\varepsilon, x+\varepsilon]$. Функция $\omega(\varepsilon) \rightarrow 0$ при $\varepsilon \rightarrow 0$, так как функция $h(x) \in C[x-\varepsilon, x+\varepsilon]$. В силу произвольности выбора $\varepsilon$ и оценок (26) и $(27)$ легко заметить, что второе слагаемое в (25) тоже стремится к нулю.

Преобразуем интеграл $J_{2}(x, t)$. Для этого, учитывая $(4)$ и $(18)$, запишем

$$
\begin{aligned}
\lim _{t \rightarrow 0} J_{2}(x, t) & = \\
& =\frac{|x|^{\beta-1 / 2} h(x)}{2 \sqrt{\pi}} \lim _{t \rightarrow 0}\left[\int_{x_{1}}^{x} \frac{|\xi|^{1 / 2-\beta}}{\sqrt{t}} \exp \left(-\frac{(x-\xi)^{2}}{4 t}\right) d \xi+\right. \\
& \left.+\int_{x}^{x_{2}} \frac{|\xi|^{1 / 2-\beta}}{\sqrt{t}} \exp \left(-\frac{(x-\xi)^{2}}{4 t}\right) d \xi\right] .
\end{aligned}
$$

В последних двух интегралах сделаем замену переменной интегрирования по формулам $\xi=x-2 \sqrt{t} s$ и $\xi=x+2 \sqrt{t} s$ соответственно. В результате будем иметь

$$
\begin{aligned}
& \lim _{t \rightarrow 0} J_{2}(x, t)= \\
& \quad=\frac{|x|^{\beta-1 / 2} h(x)}{\sqrt{\pi}} \lim _{t \rightarrow 0}\left[\int_{0}^{\frac{x-x_{1}}{2 \sqrt{t}}} \frac{e^{-s^{2}} d s}{|x-2 \sqrt{t} s|^{\beta-1 / 2}}+\int_{0}^{\frac{x_{2}-x}{2 \sqrt{t}}} \frac{e^{-s^{2}} d s}{|x+2 \sqrt{t} s|^{\beta-1 / 2}}\right] .
\end{aligned}
$$

Переходя к пределу в последнем равенстве и учитывая известную формулу

$$
2 \int_{0}^{+\infty} e^{-s^{2}} d s=\sqrt{\pi}
$$


получим:

$$
\lim _{\eta \rightarrow y} \int_{x_{1}}^{x_{2}}|\xi|^{b} h(\xi) D_{y \eta}^{\alpha-1} \Gamma(x, \xi, y-\eta) d \xi=\lim _{t \rightarrow 0} J_{2}(x, t)=h(x) .
$$

Из свойств $3^{\circ}$ и $4^{\circ}$ следует, что функция $\Gamma(x, \xi, y)$, определяемая равенством (17), является фундаментальным решением уравнения (1).

При $\beta=1 / 2(b=0)$ из $(9),(17)$ и (18) следует

$$
\Gamma(x, \xi, y-\eta)=\left.A_{t}^{\alpha} g(x, \xi, t)\right|_{t=y-\eta}, \quad g(x, \xi, t)=\frac{1}{2 \sqrt{\pi t}} \exp \left(-\frac{(x-\xi)^{2}}{4 t}\right) .
$$

Учитывая (10), последнее равенство можно записать в виде

$$
g(x, \xi, t)=\frac{1}{2 \sqrt{t}} \phi\left(-\frac{1}{2}, \frac{1}{2} ;-\frac{|x-\xi|}{\sqrt{t}}\right) .
$$

Тогда, используя формулу (16) при $\mu=0, \delta=1 / 2, \rho=-1 / 2$ и $c=|x-\xi|$, получим функцию

$$
\Gamma(x, \xi, y-\eta)=\frac{(y-\eta)^{\sigma-1}}{2} \phi\left(-\sigma, \sigma ;-\frac{|x-\xi|}{(y-\eta)^{\sigma}}\right), \quad \sigma=\frac{\alpha}{2},
$$

которая совпадает с фундаментальным решением уравнения диффузии дробного порядка, приведенным в [2, с. 127].

4. Общее представление решения. Имеет место следующая теорема об общем представлении решения уравнения (1).

Tеорема. Пусть $y^{1-\alpha} f(x, y) \in C(\bar{\Omega}), \varphi(x) \in C\left[r_{1} ; r_{2}\right]$, а функиия $v=$ $=v(x, y ; \xi, \eta)$ удовлетворяет следующим условиям:

1) в области $\Omega_{y} \backslash\{\xi=0\}$ функиия $v$ является решением уравнения

$$
\mathbf{L}^{*} v(x, y ; \xi, \eta)=q(x, y ; \xi, \eta)
$$

где $\eta^{1-\alpha} q \in L\left(\Omega_{y}\right)$

2) для любой функиии $h(x) \in C\left[x_{1} ; x_{2}\right], r_{1} \leqslant x_{1}<x_{2} \leqslant r_{2}$, выполняется соотношение

$$
\lim _{\eta \rightarrow y} \int_{x_{1}}^{x_{2}}|\xi|^{b} h(\xi) D_{y \eta}^{\alpha-1} v(x, y ; \xi, \eta) d \xi=h(x), \quad x_{1}<x<x_{2}
$$

3) функиия $v$ непрерывна в $\bar{\Omega} \times \bar{\Omega}_{y} \backslash\{y=\eta\}$ вместе с $|\xi|^{b} v_{\xi}, D_{y \eta}^{\alpha} v$ u $y^{1-\alpha} v$, и для любых точек $(x, y) \in \Omega u(\xi, \eta) \in \Omega_{y}$ выполняется неравенство

$$
|v(x, y ; \xi, \eta)| \leqslant \text { const } \cdot(y-\eta)^{\alpha \beta-1} \text {. }
$$

Если функиия $и(x, y)$ является регулярным решением уравнения (1), имеет непрерьвную и интегрируемую производную с весом $|x|^{b} u_{x}(x, y)$ вплоть до участков границы $x=r_{1} u x=r_{2}$ и удовлетворяет условию

$$
\Gamma(\alpha) \lim _{y \rightarrow 0} y^{1-\alpha} u(x, y)=\varphi(x), \quad r_{1}<x<r_{2},
$$


то для любой точки $(x, y) \in \Omega$ имеет место соотношение

$$
\begin{aligned}
u(x, y)= & \int_{r_{1}}^{r_{2}}|\xi|^{b} v(x, y ; \xi, 0) \varphi(\xi) d \xi+ \\
& +\int_{0}^{y}\left[\left|r_{2}\right|^{b} v\left(x, y ; r_{2}, \eta\right) u_{\xi}\left(r_{2}, \eta\right)-\left|r_{1}\right|^{b} v\left(x, y ; r_{1}, \eta\right) u_{\xi}\left(r_{1}, \eta\right)-\right. \\
& \left.-\left|r_{2}\right|^{b} v_{\xi}\left(x, y ; r_{2}, \eta\right) u\left(r_{2}, \eta\right)+\left|r_{1}\right|^{b} v_{\xi}\left(x, y ; r_{1}, \eta\right) u\left(r_{1}, \eta\right)\right] d \eta+ \\
& \quad+\int_{r_{1}}^{r_{2}} \int_{0}^{y}|\xi|^{b}[u(\xi, \eta) q(x, y ; \xi, \eta)-v(x, y ; \xi, \eta) f(\xi, \eta)] d \eta d \xi
\end{aligned}
$$

Предварительно, воспользовавшись оператором

$$
\left(S_{s t}^{\delta} g\right)(\eta) \equiv S_{s t}^{\delta} g(\eta)=\frac{\operatorname{sign}(t-s)}{\pi} \int_{s}^{t} \frac{g(\xi)}{\xi-\eta}\left|\frac{\xi-s}{s-\eta}\right|^{\delta} d \xi
$$

приведем лемму [2, с. 118], которая понадобится при доказательстве теоремы.

Лемма. Пусть $0 \leqslant \eta<y_{\varepsilon}<y, y_{\varepsilon}=y-\varepsilon$. Если

$$
g(\eta) \leqslant \operatorname{const} \cdot(y-\eta)^{\alpha \beta-1}
$$

то для любого $\theta \in[0 ; 1]$ справедлива оценка

$$
\left|S_{y_{\varepsilon} y}^{\delta} g(\eta)\right| \leqslant \text { const } \cdot \varepsilon^{\alpha \beta+\delta-\theta}\left(y_{\varepsilon}-\eta\right)^{\theta-\delta-1} .
$$

Доказательств о те о рем и. Рассмотрим выражение

$$
\int_{r_{1}}^{r_{2}} \int_{0}^{y_{\varepsilon}}|\xi|^{b}\left[v(x, y ; \xi, \eta) \mathbf{L} u(\xi, \eta)-u(\xi, \eta) \mathbf{L}^{*} v(x, y ; \xi, \eta)\right] d \eta d \xi
$$

где $y_{\varepsilon}=y-\varepsilon, \varepsilon>0$.

В работе [2, с. 121] доказано равенство

$$
\begin{aligned}
\int_{0}^{y_{\varepsilon}}\left(v D_{0 \eta}^{\alpha} u-u D_{y \eta}^{\alpha} v\right) d \eta=u\left(\xi, y_{\varepsilon}\right) & D_{y y_{\varepsilon}}^{\alpha-1} v\left(x, y ; \xi, y_{\varepsilon}\right)- \\
& -\varphi(\xi) v(x, y ; \xi, 0)+R\left(x, y, y_{\varepsilon}, \xi\right),
\end{aligned}
$$

где

$$
R\left(x, y, y_{\varepsilon}, \xi\right)=\sin \pi(\alpha-1) \cdot \int_{0}^{y_{\varepsilon}} S_{y_{\varepsilon} y}^{1-\alpha} v \cdot D_{0 \eta}^{\alpha} u d \eta-\left.\sin \pi \alpha \cdot \varphi(\xi) S_{y_{\varepsilon} y}^{1-\alpha} v\right|_{\eta=0} .
$$

Учитывая (32) и соотношение

$$
v \frac{\partial}{\partial \xi}\left(|\xi|^{b} \frac{\partial u}{\partial \xi}\right)-u \frac{\partial}{\partial \xi}\left(|\xi|^{b} \frac{\partial v}{\partial \xi}\right)=\frac{\partial}{\partial \xi}\left(|\xi|^{b} v \frac{\partial u}{\partial \xi}-|\xi|^{b} u \frac{\partial v}{\partial \xi}\right)
$$


преобразуем (31):

$$
\begin{gathered}
\int_{r_{1}}^{r_{2}} \int_{0}^{y_{\varepsilon}}|\xi|^{b}\left[v(x, y ; \xi, \eta) \mathbf{L} u(\xi, \eta)-u(\xi, \eta) \mathbf{L}^{*} v(x, y ; \xi, \eta)\right] d \eta d \xi= \\
=\left.\int_{0}^{y_{\varepsilon}}|\xi|^{b}\left[v(x, y ; \xi, \eta) u_{\xi}(\xi, \eta)-v_{\xi}(x, y ; \xi, \eta) u(\xi, \eta)\right]\right|_{\xi=r_{1}} ^{\xi=r_{2}} d \eta+ \\
+\int_{r_{1}}^{r_{2}}|\xi|^{b}\left[v(x, y ; \xi, 0) \varphi(\xi)-u\left(\xi, y_{\varepsilon}\right) D_{y y_{\varepsilon}}^{\alpha-1} v\left(x, y ; \xi, y_{\varepsilon}\right)-R\left(x, y, y_{\varepsilon}, \xi\right)\right] d \xi
\end{gathered}
$$

Из выше приведенной леммы следует, что

$$
\lim _{\varepsilon \rightarrow 0} \int_{r_{1}}^{r_{2}}|\xi|^{b} R\left(x, y, y_{\varepsilon}, \xi\right) d \xi=0
$$

Поэтому из (1), (28), (29) и (33), устремляя $\varepsilon$ к нулю, получим соотношение $(30)$.

Заключение. В работе в терминах интегрального преобразования с функцией Райта в ядре построено и исследованы свойства фундаментального решения модельного уравнения аномальной диффузии дробного порядка, которое может быть использовано при моделировании задач переноса в средах с фрактальной структурой. Показано, что когда исследуемое уравнение переходит в уравнение диффузии дробного порядка, построенное фундаментальное решение переходит в фундаментальное решение соответствующего уравнения. Построено общее представление решения рассматриваемого уравнения в прямоугольной области.

\section{ORCID}

Фатима Гидовна Хуштова: http://orcid.org/0000-0003-4088-3621

\section{БИБЛИОГРАФИЧЕСКИЙ СПИСОК}

1. Нахушев А. М. Уравнения математической биологии. М.: Высшая школа, 1995. 301 с.

2. Псху А. В. Уравнения в частных производных дробного порядка. М.: Наука, 2005. 199 с.

3. Терсенов С. А. Параболические уравнения с меняющимся направлением времени. М.: Наука, 1985. 105 с.

4. Pagani C. On the parabolic equation $\operatorname{sgn}(x)|x|^{p} u_{y}-u_{x x}=0$ and a related one // Annali di Matematica Pura ed Applicata, Series 4, 1974. vol.99, no. 1. pp. 333-339. doi: 10.1007/ BF02413730.

5. Kȩpiński M. S. Integration der Differentialgleichung $\frac{\partial^{2} j}{\partial \xi^{2}}-\frac{1}{\xi} \frac{\partial j}{\partial t}=0 /$ Krakau Anz., 1905. pp. 198-205.

6. Arena O. On a degenerate elliptic-parabolic equation// Communications in Partial Differential Equations, 1978. vol.11, no.3. pp. 1007-1040. doi:10.1080/ 03605307808820084.

7. Mainardi F. The time fractional diffusion-wave equation// Radiophysics and Quantum Electronics, 1995. vol. 38, no.1-2. pp. 13-24. doi : 10.1007/bf01051854.

8. Mainardi F. The fundamental solutions for the fractional diffusion-wave equation // Applied Mathematics Letters, 1996. vol. 9, no. 6. pp. 23-28. doi : 10.1016/0893-9659(96)00089-4.

9. Псху А. В. Фундаментальное решение диффузионно-волнового уравнения дробного порядка // Изв. РАН. Сер. матем., 2009. Т. 73, № 2. С. 141-182. doi: 10.4213/im2429. 
10. Ворошилов А. А., Килбас А. А. Задача типа Коши для диффузионно-волнового уравнения с частной производной Римана-Лиувилля // Докладь Академии наук, 2006. Т. 406, № 1. C. $12-16$.

11. Геккиева С. Х. Задача Коши для обобщенного уравнения переноса с дробной по времени производной // Доклады Адыгской (Черкесской) Международной академии наук, 2000. Т. 5, № 1. С. 16-19.

12. Кочубей А. Н. Задача Коши для эволюционных уравнений дробного порядка // Дифферени. уравнения, 1989. Т. 25, № 8. С. 1359-1368.

13. Кочубей А. Н. Диффузия дробного порядка // Дифферени. уравнения, 1990. Т. 26 , № 4. C. 660-670.

14. Metzler R., Glöckle W. G., Nonnenmacher T. F. Fractional model equation for anomalous diffusion// Physica A: Statistical Mechanics and its Applications, 1994. vol.211, no.1. pp. 13-24. doi: 10.1016/0378-4371(94)90064-7.

15. Giona M., Roman H. E. Fractional diffusion equation on fractals: one-dimensional case and asymptotic behavior // Phys. A: Math. Gen., 1992. vol. 25, no. 8. pp. 2093-2105. doi: 10. 1088/0305-4470/25/8/023.

16. Metzler R., Klafter J. The random walk's guide to anomalous diffusion: a fractional dynamics approach // Physics Reports, 2000. vol.339, no.1. pp. 1-77. doi: 10.1016/s0370-1573(00) 00070-3.

17. Metzler R., Klafter J. The restaurant at the end of the random walk: recent developments in the description of anomalous transport by fractional dynamics // Phys. A: Math. Gen., 2004. vol. 37, no. 31. pp. R161-R208. doi : 10.1088/0305-4470/37/31/r01.

18. Учайкин В. В. Анизотропия космических лучей в дробно-дифференциальных моделях аномальной диффузии // ЖЭТФ, 2013. Т. 143, №6. С. 1039-1047. doi: 10.7868/ S0044451013060037.

19. Uchaikin V. V. Fractional Derivatives for Physicists and Engineers / Nonlinear Physical Science. vol. I: Background and Theory. Berlin: Springer, 2013. xii+385 pp. doi: 10.1007/ 978-3-642-33911-0.

20. Кузнецов Д. С. Специалъные функиии. М.: Высшая школа, 1962. 248 с.

21. Erdíyi A., Magnus W., Oberhettinger F., Tricomi F. G. Higher transcendental functions. vol. II. / Bateman Manuscript Project. New York, Toronto, London: McGraw-Hill Book Co., 1953. xvii+396 pp.

22. Wright E. M. On the coefficients of power series having exponential singularities // J. London Math. Soc., 1933. vol.s1-8, no. 1. pp. 71-79. doi: 10.1112/jlms/s1-8.1.71.

23. Gorenflo R., Luchko Y., Mainardi F. Analytical properties and applications of the Wright function // Fractional Calculus and Applied Analysis, 1999. vol. 2, no. 4. pp. 383-414, arXiv: math-ph/0701069.

Поступила в редакцию 15/VIII/2015; в окончательном варианте - 19/X/2015; принята в печать - 19/XI/2015. 
Vestn. Samar. Gos. Techn. Un-ta. Ser. Fiz.-mat. nauki

[J. Samara State Tech. Univ., Ser. Phys. \& Math. Sci.], 2015, vol. 19, no. 4, pp. $722-735$

ISSN: 2310-7081 (online), 1991-8615 (print)

doi: http://dx.doi.org/10.14498/vsgtu1445

MSC: 35A08, 35A22, 35R11, 35C15

\title{
FUNDAMENTAL SOLUTION OF THE MODEL EQUATION OF ANOMALOUS DIFFUSION OF FRACTIONAL ORDER
}

\section{F. G. Khushtova}

Institution of Applied Mathematics and Automation, 89 a, Shortanova st., Nal'chik, 360000, Russian Federation.

\begin{abstract}
Fundamental solution of the model equation of anomalous diffusion with Riemann-Liouville operator is constructed. Using the properties of the integral transformation with Wright function in kernel, we give estimates for the fundamental solution. When the considered equation transformes into the diffusion equation of fractional order, constructed fundamental solution goes into the corresponding fundamental solution of the diffusion equation of fractional order. General solution of the model equation of anomalous diffusion of fractional order is constructed.
\end{abstract}

Keywords: anomalous diffusion, diffusion fractional order, Riemann-Liouville operator, fundamental solution, general representation of solution, modified Bessel function, Wright function, integral transformation wich Wright function in kernel.

doi: http://dx.doi.org/10.14498/vsgtu1445

\section{ORCID}

Fatima G. Khushtova: http://orcid.org/0000-0003-4088-3621

\section{REFERENCES}

1. Nakhushev A. M. Uravneniia matematicheskoi biologii [Equations of mathematical biology]. Moscow, Vysshaia shkola, 1995, 301 pp. (In Russian)

2. Pskhu A. B. Uravneniia $v$ chastnykh proizvodnykh drobnogo poriadka [Partial differential equations of fractional order]. Moscow, Nauka, 2005, 199 pp. (In Russian)

3. Tersenov S. A. Parabolicheskie uravneniia s meniaiushchimsia napravleniem vremeni [Parabolic equations with varying time direction]. Moscow, Nauka, 1985, 105 pp. (In Russian)

4. Pagani C. On the parabolic equation $\operatorname{sgn}(x)|x|^{p} u_{y}-u_{x x}=0$ and a related one, Annali di Matematica Pura ed Applicata, Series 4, 1974, vol.99, no.1, pp. 333-339. doi: 10.1007/ BF02413730.

(C) 2015 Samara State Technical University.

Please cite this article in press as:

Khushtova F. G. Fundamental solution of the model equation of anomalous diffusion of fractional order, Vestn. Samar. Gos. Tekhn. Univ., Ser. Fiz.-Mat. Nauki [J. Samara State Tech. Univ., Ser. Phys. \& Math. Sci.], 2015, vol. 19, no. 4, pp. 722-735. doi: 10.14498/vsgtu1445. (In Russian)

Author Details:

Fatima G. Khushtova (khushtova@yandex.ru), Research Fellow, Dept. CAD of Mixed Systems and Management. 
5. Kȩpiński M. S. Integration der Differentialgleichung $\frac{\partial^{2} j}{\partial \xi^{2}}-\frac{1}{\xi} \frac{\partial j}{\partial t}=0$, Krakau Anz., 1905, pp. 198-205.

6. Arena O. On a degenerate elliptic-parabolic equation, Communications in Partial Differential Equations, 1978, vol.11, no.3, pp. 1007-1040. doi:10.1080/ 03605307808820084.

7. Mainardi F. The time fractional diffusion-wave equation, Radiophysics and Quantum Electronics, 1995, vol. 38, no. 1-2, pp. 13-24. doi: 10.1007/bf01051854.

8. Mainardi F. The fundamental solutions for the fractional diffusion-wave equation, Applied Mathematics Letters, 1996, vol. 9, no. 6, pp. 23-28. doi : 10.1016/0893-9659(96)00089-4.

9. Pskhu A. V. The fundamental solution of a diffusion-wave equation of fractional order, Izv. Math., 2009, vol. 73, no. 2, pp. 351-392. doi: 10.1070/IM2009v073n02ABEH002450.

10. Voroshilov A. A., Kilbas A. A. A Cauchy-type problem for the diffusion-wave equation with Riemann-Liouville partial derivative, Dokl. Math., 2006, vol.73, no. 1, pp. 6-10. doi: 10 . 1134/S1064562406010029.

11. Gekkieva S. H. The Cauchy problem for the generalized transmission equation with a fractional derivative with respect to the time, Doklady Adygskoi (Cherkesskoi) Mezhdunarodnoi akademii nauk, 2000, vol. 5, no. 1, pp. 16-19 (In Russian).

12. Kochubej A. N. A Cauchy problem for evolution equations of fractional order, Differ. Equations, 1989, vol. 25, no. 8, pp. 967-974.

13. Kochubei A. N. Fractional-order diffusion, Differ. Equations, 1990, vol. 26, no. 4, pp. 485492.

14. Metzler R., Glöckle W. G., Nonnenmacher T. F. Fractional model equation for anomalous diffusion, Physica A: Statistical Mechanics and its Applications, 1994, vol. 211, no. 1, pp. 1324. doi : 10.1016/0378-4371(94)90064-7.

15. Giona M., Roman H. E. Fractional diffusion equation on fractals: one-dimensional case and asymptotic behavior, Phys. A: Math. Gen., 1992, vol.25, no. 8, pp. 2093-2105. doi: 10. 1088/0305-4470/25/8/023.

16. Metzler R., Klafter J. The random walk's guide to anomalous diffusion: a fractional dynamics approach, Physics Reports, 2000, vol.339, no.1, pp. 1-77. doi:10.1016/s0370-1573(00) 00070-3.

17. Metzler R., Klafter J. The restaurant at the end of the random walk: recent developments in the description of anomalous transport by fractional dynamics, Phys. A: Math. Gen., 2004, vol. 37, no. 31, pp. R161-R208. doi: 10.1088/0305-4470/37/31/r01.

18. Uchaikin V. V. Cosmic ray anisotropy in fractional differential models of anomalous diffusion, JETP, 2013, vol.116, no.6, pp. 897-903. doi: 10.1134/S1063776113050269.

19. Uchaikin V. V. Fractional Derivatives for Physicists and Engineers, Nonlinear Physical Science, vol. I, Background and Theory. Berlin, Springer, 2013. xii+385 pp. doi: 10.1007/ 978-3-642-33911-0.

20. Kuznetsov D. S. Spetsial'nye funktsii [Special functions]. Moscow, Vysshaia shkola, 1962, 248 pp. (In Russian)

21. Erdĺyi A., Magnus W., Oberhettinger F., Tricomi F. G. Higher transcendental functions, vol. II., Bateman Manuscript Project. New York, Toronto, London, McGraw-Hill Book Co., 1953, xvii+396 pp.

22. Wright E. M. On the coefficients of power series having exponential singularities, J. London Math. Soc., 1933, vol.s1-8, no. 1, pp. 71-79. doi: 10.1112/jlms/s1-8.1.71.

23. Gorenflo R., Luchko Y., Mainardi F. Analytical properties and applications of the Wright function, Fractional Calculus and Applied Analysis, 1999, vol. 2, no. 4, pp. 383-414, arXiv: math-ph/0701069.

Received 15/VIII/2015; received in revised form $19 / \mathrm{X} / 2015$; accepted 19/XI/2015. 